VOL. $6(1972), 357-378$.

\title{
The skeleton of a variety of groups
}

\section{R.M. Bryant and L.G. Kovács}

The skeleton $S(\underline{\underline{U}})$ of a variety $\underline{\underline{U}}$ of groups is defined to be the intersection of the section closed classes of groups which generate $\underline{\underline{\mathrm{U}}}$. If $m$ is an integer, $m>1, \underline{A}_{m}$ is the variety of all abelian groups of exponent dividing $m$, and $\underline{\underline{v}}$ is any locally finite variety, it is shown that the skeleton of the product variety $A \mathrm{~V}$ is the section closure of the class of finite monolithic groups in $\stackrel{\mathrm{A}}{\rightarrow} \mathrm{V}$. In particular, $S(A, \mathrm{~V}$ generates $A \mathrm{~A}$. The elements of $S\left(\mathrm{~A}_{m} \mathrm{~V}\right)$ are described more explicitly and as a consequence it is shown that $S(A, V)$ consists of all finite groups in $\underset{m}{\mathrm{~A}}$ if and only if $m$ is a power of some prime $p$ and the centre of the countably infinite relatively free group of $\underline{V}$ is a p-group.

\section{Introduction}

If $D$ is a class of groups then $S D$ and $Q D$ denote the classes of all groups isomorphic to, respectively, subgroups and factor groups of groups in D. If $G$ is a group, an element of $Q s\{G\}$ will be called a section of $G$. A class $\underline{\underline{D}}$ is called section closed if $Q \mathrm{SD}=\underline{\underline{D}}$ and, as is well-known, for any class $D, Q S D$ is section closed. For basic facts and terminology relating to varieties of groups we refer to [9] - where, however, a section is called a factor. For any variety $\underline{\underline{U}}$ we write $F(\underline{U})$ for the class of all finite groups in $\underline{\underline{U}}, M(\underline{\underline{U}})$ for the class of all finite monolithic groups in $\mathrm{U}$ and $\mathcal{C}(\underline{U})$ for the class of all critical

Received 7 December 1971. The authors are indebted to Mr L.F. Harris and $\mathrm{Dr}$ M.F. Newman for the interest they showed while this work was in progress, and for several useful suggestions. 
groups in $\underline{\underline{U}}$. Thus

$$
\mathcal{C}(\underline{\underline{U}}) \subseteq M(\underline{\underline{U}}) \subseteq F(\underline{\underline{U}})
$$

A denotes the variety of all abelian groups and, for any positive integer $m$, A the variety of all abelian groups of exponent dividing $m$. Also, $C(m)$ denotes a cyclic group of order $m$.

The skeleton of a variety $\underline{\underline{U}}$ is denoted by $S(\underline{\underline{U}})$ and is defined to be the intersection of the section closed classes of groups which generate $\underline{\underline{U}}$. Thus $S(\underline{\mathrm{U}})$ consists of the groups $G$ with the property that $G \in Q \mathrm{SD}$ whenever $\operatorname{varD}=\underline{\underline{U}}$. We shall be interested in varieties $\underline{U}$ such that $S(\underline{\underline{U}})$ generates $\underline{\underline{U}}$ and which have, therefore, a unique minimal section closed generating class. Not all varieties have this property: neither do all locally finite varieties, nor all product varieties. We note the following examples.

(i) $S(\underline{\underline{A}})$ consists only of groups of order 1 .

(ii) If $\underline{U}$ is the (locally finite) variety generated by the dihedral group of order 8 then

$$
S(\underline{\underline{U}})=\operatorname{Qs}\{C(4), C(2) \times C(2)\} \text {. }
$$

(iii) For any prime $p, S(\underline{A A})=Q s\{C(p)\}$ and $S\left(\underline{A}_{p} \underline{A}\right)=F\left(\underline{A}_{p}\right)$.

If $\underline{U}$ is a locally finite variety then $\underline{\underline{U}}=. \operatorname{var} C(\underline{\underline{U}})$, by 51.41 of [9]. Thus $S(\underline{\underline{U}}) \subseteq Q s C(\underline{\underline{U}})$. If also $C(\underline{\underline{U}}) \subseteq S(\underline{\underline{U}})$ then $S(\underline{\underline{U}})=Q \mathrm{SC}(\underline{\underline{U}})$ and $S(\underline{U})$ generates $\underline{U}$. Certain locally finite varieties are known to have this property. For example, Theorems 2 and 3 of Cossey [4] show that if $\mathrm{U}$ is a variety of $A$-groups (a locally finite variety in which nilpotent groups are abelian) then $\mathcal{C}(\underline{\underline{U}}) \subseteq S(\underline{\underline{U}})$. Also, in this case, $\mathcal{C}(\underline{\underline{U}})=M(\underline{\underline{U}})$, by (1.66) of [8]. Thus, if $\underline{U}$ is a variety of A-groups,

$$
S(\underline{\underline{U}})=\operatorname{QSC}(\underline{\underline{U}})=Q \Delta M(\underline{\underline{U}}) \text {. }
$$

One of the objects of the present paper is to prove a similar result for product varieties of the form $\mathrm{AV}$. where $m$ is a positive integer, $m>1$, and $\underline{V}$ is a locally finite variety. $A \mathrm{~V}$ is locally finite by 21.14 of [9]. It is a consequence of the Corollary of Brisley and Kovács

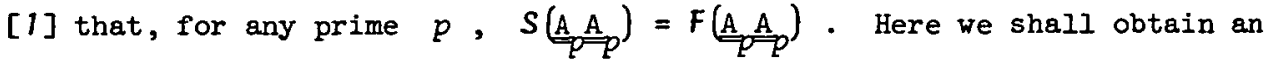


explicit description of the elements of $S(A, V)$ and, in particular, extend the Brisley-Kovács result by finding necessary and sufficient conditions on $m$ and $\underline{\underline{V}}$ to ensure $S(\underline{A} \underline{\underline{A}})=F(\underline{A}, \underline{V})$. Such locally finite varieties $\underline{\underline{U}}$ satisfying $S(\underline{\underline{U}})=F(\underline{\underline{U}})$ are particularly interesting. They have the property that every class of groups which generates $\underline{\underline{U}}$ discriminates $\underline{\underline{U}}$. (See Chapter 1, $\$ 7$, of [9] for the notion of discrimination.) This is a consequence of the following remark (proof below).

(1.1). Let $\mathrm{D}$ be a class of groups contained in the locally finite variety . Then $\underline{\underline{D}}$ discriminates $\underline{\underline{\mathrm{U}}}$ if and only if $F(\underline{\underline{\mathrm{U}}}) \subseteq \mathrm{QSD}$.

Before we state our main results we fix some notation. $X_{\infty}$ denotes an absolutely free group freely generated by elements $x_{1}, x_{2}, \ldots$, and we write $X_{k}$ for the subgroup $\left\langle x_{1}, x_{2}, \ldots, x_{k}\right\rangle$ of $X_{\infty}$ generated by $x_{1}, x_{2}, \ldots, x_{k}$ : this is a free group of rank $k$. If $\underline{\underline{V}}$ is a variety we write $F_{k}(\underline{\underline{v}})$ for the relatively free group of $\underline{\underline{\mathrm{V}}}$ of finite rank $k$ and $F_{\infty}(\underline{Y})$ for the relatively free group of countably infinite rank. $\underline{\underline{V}}(G)$ denotes the verbal subgroup of a group $G$ corresponding to $\underline{v}$. We shall always take $p$ to be a prime. If $G$ is a group then $z(G)$ denotes the centre of $G$ and $O_{p}, Z(G)$ the subgroup of $Z(G)$ consisting of the elements of finite order prime to $p$. For any variety $\underline{V}, o_{p^{\prime}} z\left(F_{\infty}(\underline{V})\right)$ is a fully-invariant subgroup of $F_{\infty}(\underline{V})$. We write

$$
\stackrel{\mathrm{V}}{\mathrm{p}}_{\mathrm{p}}=\operatorname{var} F_{\infty}(\underline{\mathrm{V}}) / 0_{p^{\prime}} Z\left(F_{\infty}(\underline{\mathrm{V}})\right) .
$$

THEOREM 1.2. For any locally finite variety $\mathrm{V}$ and any integer $m>1$,

$$
S(\underline{A}, V)=\operatorname{asC}(\underline{A}, V)=\operatorname{asM}(\underline{A}, V)
$$

Hence $S(\mathrm{~A}, \mathrm{~V})$ generates $\mathrm{A} \mathrm{V}$.

Suppose that $m=p(1)^{\alpha(1)} \ldots p(r)^{\alpha(r)}$ where the $p(i)$ are distinct primes, the $\alpha(i)$ are positive integers, and $r \geq 1$. Then, for any locally finite variety $\underline{v}$, 


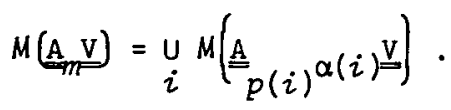

Thus, by Theorem 1.2,

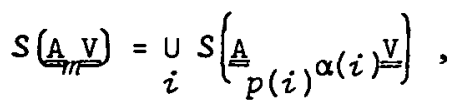

and to determine the elements of $S(A, V)$ it suffices to consider the case where $m$ is a prime power. This is done in the next two results.

(1.3). Let $n$ and $\alpha$ be positive integers, $n$ prime to $p$. Then

$$
s\left(\underline{A}_{p} \alpha \underline{A}\right)=\operatorname{Qs}\left\{C\left(p^{\alpha}, n\right)\right\} \text {, }
$$

where $c\left(p^{\alpha}, n\right)$ is the unique critical group which generates ${ }_{p}^{\mathrm{A}} \alpha^{\mathrm{A}_{n}}$.

(1.3) covers the exceptional case to

THEOREM 1.4. Let $\mathrm{V}$ be a locally finite variety which is not abelian of exponent prime to $p$, and let $\alpha$ be a positive integer. Then $S\left(\underline{\underline{A}}_{p} \alpha\right)$ consists of all groups $G$ of $F\left(\underline{\underline{A}}_{p} \alpha \underline{v}\right)$ such that $\underline{\underline{V}}_{p}(G) / \underline{\underline{V}}(G)$ is cyclic and, if $\underline{\mathrm{V}}(G)$ is regarded as a $\stackrel{\mathrm{V}}{p}_{p}(G) / \mathrm{V}(G)$-module, its composition factors (if any) are isomorphic and faithful.

COROLLARY 1.5. Let $\underline{\mathrm{V}}$ be a locally finite variety and $m a$ positive integer, $m>1$. Then $S\left(\mathrm{~A}_{m} \mathrm{~V}\right)=F(\mathrm{~A}, \mathrm{~V})$ if and only if $m$ is a power of some prime $p$ and $z\left(E_{\infty}(\underline{V})\right)$ is a p-group.

We conclude this section by giving the proofs of (1.1) and (1.3) and the derivation of Corollary 1.5 from Theorems 1.2 and 1.4 . The proofs of Theorems 1.2 and 1.4 will be completed in $\$ 4$. $\$ \S 2$ and 3 will be devoted to some preliminary results. However, of these, only (2.1) to (2.5) are needed for the proof of Theorem 1.2.

Proof of (1.1). Let $D$ and $\mathrm{U}$ be as in the statement of (1.1). If $F(\underline{U}) \subseteq Q 8 D$ then $\underline{D}$ discriminates $\underline{U}$ by 17.41 and 17.5 of [9]. Conversely, suppose that $\underline{D}$ discriminates $\underline{\underline{U}}$. Let $\underline{\underline{W}}$ be a set of representatives of the non-trivial cosets of $\mathrm{U}\left(X_{n}\right)$ in $X_{n}$. Then $\mathrm{w}$ is 
finite since $X_{n} / \underline{\underline{U}}\left(X_{n}\right) \cong F_{n}(\underline{\underline{U}})$ is finite. Thus $\underline{\underline{w}}$ is a finite set of non-laws of $\underline{\underline{\mathrm{U}}}$ and there is a group $D \in \underline{\underline{D}}$ and a homomorphism $\theta: X_{n} \rightarrow D$ such that $w \theta \neq 1$. for all $w \in \underline{W}$. Since $D \in \underline{\underline{U}}, \underline{\underline{U}}\left(X_{n}\right) \leq \operatorname{ker} \theta$, and it follows that $\underline{\underline{U}}\left(X_{n}\right)=\operatorname{ker} \theta$. Therefore $F_{n}(\underline{\underline{U}}) \in \mathrm{sD}$. But every element of $F(\underline{\underline{U}})$ lies in $Q\left\{F_{n}(\underline{\underline{U}})\right\}$ for some $n$. Thus $F(\underline{\underline{U}}) \subseteq Q s \underline{\underline{D}}$.

Proof of (1.3). In Chapter 4 of Cossey [3] it is shown that if $n$ is prime to $p$ then there is a unique critical group $C\left(p^{\alpha}, n\right)$ which generates $\underset{p}{\stackrel{A}{A}} \underset{p}{A}$. (This group has a normal subgroup which is a direct product of $t$ copies of $\dot{c}\left(p^{\alpha}\right)$, where $t$ is the least positive integer such that $n \mid p^{t}-1$, and with factor group $c(n)$. ) By Theorem 3 of [4] (or by Theorem 1.2), $c\left(p^{\alpha}, n\right) \in s\left(\underline{\underline{\underline{A}}} \alpha_{p}^{\alpha}\right)$ and it follows that

$$
s\left(\underline{\underline{A}}_{p} \alpha \underline{\underline{A}}\right)=\operatorname{as}\left\{C\left(p^{\alpha}, n\right)\right\} \text {. }
$$

Derivation of Corollary 1.5. If $m$ is a power of $p$ and $z\left(F_{\infty}(\underline{\underline{V}})\right)$ is a $p$-group, then $\underline{\underline{V}}=\frac{\mathrm{V}}{p}$ and so $F(\underline{A} \underline{\mathrm{V}})=S(\underline{\mathrm{A}} \mathrm{V})$ by Theorem 1.4. To see the converse, first note that the order of $v\left(E_{1}\left(A_{m} v\right)\right)$ is $m$, while Theorem 1.2 implies that $\underline{\underline{\mathrm{V}}}(H)$ has prime-power order whenever $\theta \in S(\underline{A} \mathrm{~V})$. Thus, if $F(A, V)=S(A, V)$ then $m$ is a prime power - say a power of $p$. In this case, if $\underline{\mathrm{V}}_{p}(G)$ were non-trivial for some $G \in F(\underline{\mathrm{V}})$ then $\mathrm{V}_{p}(G \times G)$ would be non-cyclic, and, by Theorem 1.4, this would contradict

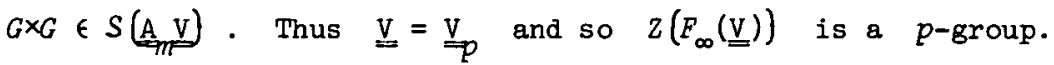

\section{Representation theory}

Most of our terminology for representation theory follows [5], but we deal with right modules instead of left modules. Unless otherwise stated all modules are finitely generated. Groups are usually written multiplicatively, but for an abelian group we may switch to additive notation when the group is being regarded as a module. For any positive 
integer $m>1, Z(m)$ will denote the ring of integers modulo $m$. Thus $Z(p)$ is a field of $p$ elements. Throughout this section $G$ will denote a finite group and $F$ a field.

If $U$ is a $G$-module we write UsplitG for the semidirect product of the abelian group $U$ by $G$, where the action of $G$ by conjugation on $U$ is the module action. If $U$ has exponent dividing $m$ then we may regard $U$ as a $Z(m) G$-module, that is, a module for the group ring $Z(m) G$ of $G$ over $Z(m)$. We shall need a result, covered by cohomology theory, for which we have not found any really convenient explicit reference. A special case is proved in the paragraph beginning at the bottom of p. 393 of [1], and the proof there immediately generalizes to give what is needed here:

(2.1). Let $U$ be an injective $Z(m) G$-module and let $H$ be an extension of $U$ by $G$, where the action of $G$ on $U$ is the module action. Then $H \cong$ Usplit $G$.

If $U$ is a $G$-module, $C_{G}(U)$ denotes the set of elements of $G$ which act trivially on $U$. We write $H \leq G$ if $H$ is a subgroup of $G$ and $H \subseteq G$ if $H$ is a normal subgroup of $G$. We shall require the following consequence of (2.1).

(2.2). Let $H$ be an extension of the $Z(m) G$-moduze $U$ by $G$, where the action of $G$ on $U$ is the module action. Suppose that $V$ is an injective submodule of $U$ and let $N \leq H$ be maximal subject to $N \leq H$ and $V \cap N=1$. Then

$$
H / N \cong \operatorname{Vsplit}\left(G / C_{G}(V)\right)
$$

Proof. $H / N$ is an extension of a factor module $U^{\prime}$ of $U$ by a factor group $G / M$ of $G$ where $M \leq C_{G}\left(U^{\prime}\right)$ and so $U^{\prime}$ may be regarded as a $Z(m)(G / M)$-module. Since $V \cap N=1, U^{\prime}$ has a submodule $V^{\prime}$, $V^{\prime} \cong V$. Since $V^{\prime}$ is an injective $Z(m) G$-module, it is an injective $Z(m)(G / M)$-module. Thus $V^{\prime}$ is complemented in $U^{\prime}$. By the choice of $N$ it follows that $V^{\prime}=U^{\prime}$, and so, by (2.1),

$$
H / N \cong \operatorname{Vsplit}(G / M) \text {. }
$$

If $G / M$ is identified with a complement of $V^{\prime}$ in $H / N$ then 
$C_{G / M}(V) \leq H / N$ and $V^{\prime} \cap C_{G / M}(V)=1$. Thus, by the choice of $N$, $M=C_{G}(V)$.

We wish to establish some facts relating to modules over the group rings $F G$ and $Z(m) G$. Both $F$ and $Z(m)$ are examples of quasi-Frobenius rings (see $\$ 58$ of [5]), so more generally we shall consider the group ring $R G$ where $R$ is a quasi-Frobenius ring with identity. By Exercise 2(d), p. 402 of [5], $R G$ is itself a quasi-Frobenius ring. In particular $R G$ has maximum condition and minimum condition on right ideals. We shall only wish to consider finitely generated $R G$-modules. However, we have occasion to use injective hulls (see 557 of [5]) and it is not perhaps obvious that the injective hull of a finitely generated $R G$-module $U$ is again finitely generated. To see this, consider the socle of $U$, denoted by $\sigma U$ - that is, the submodule generated by the irreducible submodules of $U$. Since $\sigma U$ is completely reducible and finitely generated it is the direct sum of finitely many irreducible submodules. The injective hull of $U$ is the injective hull of $\sigma U$ and this is the direct sum of the injective hulls of the irreducible summands of $\sigma U$. It remains only to note that the injective hull of an irreducible $R G$-module is a principal indecomposable $R G$-module, and is therefore a one-generator module. This follows from (58.6), (58.12) and (58.13) of [5].

The regular $R G$-module will like the group ring be denoted by $R G$. The Jordan-Hölder Theorem, (13.7) of [5], and the Krull-Schmidt Theorem, (14.5) of [5], apply to $R G$-modules and will be used without further comment. The $R G$-module $U$ is said to be a direct summand of the $R G$-module $V$ if $V$ is isomorphic to the direct sum of $U$ and some other $R G$-module. We shall write $U \lesssim V$ if $U$ is isomorphic to a submodule of $v$. If $n$ is a non-negative integer then $U^{\oplus n}$ denotes the direct sum of $n$ copies of $U$ and is called a direct multiple of $U$ (with the convention $U^{\oplus 0}=0$ ). We say that $U$ is faithful if $C_{G}(U)=1$ : note that we do not mean that $U$ has zero annihilator in $R G$. We call $U$ homogeneous if all of its composition factors are isomorphic (without requiring that $U$ be completely reducible), and monolithic if it has a unique irreducible submodule (that is, if $\sigma U$ is irreducible). For any 
integer $n$ we put

$$
n U=\{n u: u \in U\},
$$

writing $U$ additively. Thus $n U$ is a submodule of $U$. If $H \leq G$ then $U_{H}$ denotes the restriction of $U$ to $R H$. If $H \leq G$ and $V$ is an

$F H$-module then $V^{G}$ denotes the $F G$-module induced from $V$. If $E$ is an extension field of $F$ and $U$ is an $F G$-module we write $U \otimes E=U \otimes_{F} E$ for the corresponding EG-module.

By $(56.6)$ and $(58.14)$ of [5] we have

(2.3). An RG-module is injective if and only if it is isomorphic to the direct sum of finitely many principal indecomposable RG-modules.

By $(58.12)$ of $[5]$,

(2.4). A principal indecomposable $R G$-module is monolithic.

If $U$ is an indecomposable, injective $R G$-module, and $N \leq G$, $N \leq C_{G}(U)$, then $U$ is clearly indecomposable and injective regarded as an $R(G / N)$-module. In other words, by (2.3),

(2.5). If $U$ is a principal indecomposable RG-module and $N \leq G$, $N \leq C_{G}(U)$, then $U$ regarded as an $R(G / N)$-module is a principal indecomposable $R(G / N)$-module.

The remainder of this section and the whole of the next section are only needed for the proof of Theorem 1.4 .

By $(2.3)$, an $R G$-module is injective if and only if it is a direct summand of $(R G)^{\oplus n}$ for some $n$. If $H \leq G$ and $H$ has index $k$ in $G$ then it is easy to see that $\left((R G)^{\oplus n}\right)_{H} \cong(R H)^{\oplus k n}$. Thus

(2.6). If $U$ is an injective $R G$-module and $H \leq G$ then $U_{H}$ is an injective RH-module.

(2.7). If $H \leq G$ and $V$ is a principal indecomposable RH-module then, for some principal indecomposable RG-module $U, V$ is a direct summand of $U_{H}$. 
If $\alpha$ is a positive integer, $z\left(p^{\alpha}\right) G$ is homocyclic of exponent $p^{\alpha}$ and so the same is true of every principal indecomposable $z\left(p^{\alpha}\right) G$-module. Clearly $p^{\alpha-1} Z\left(p^{\alpha}\right) G$, regarded as a $Z(p) G$-module, is isomorphic to $Z(p) G$. Thus, if $U$ is a principal indecomposable $Z\left(p^{\alpha}\right) G$-module, $p^{\alpha-1} U$ is a non-zero direct summand of $Z(p) G$. Since $U$ is monolithic by (2.4), $p^{\alpha-1} U$ is also monolithic and must be a principal indecomposable $2(p) G$-module :

(2.8). If $U$ is a principal indecomposable $z\left(p^{\alpha}\right)$ G-module then $p^{\alpha-1} U$ is a principal indecomposable $z(p) G$-module.

Suppose that $F$ has characteristic $P$. Let $H \leq O_{p^{\prime}} Z(G)$ and let $U$ be an $E G$-module. Since $H$ is a $p^{\prime}$-group, $U_{H}$ is completely reducible by Maschke's Theorem, (10.8) of [5]. Thus $U_{H}$ is the direct sum of its maximal homogeneous submodules. Each such summand is $G$-invariant since $H \leq Z(G)$. Therefore

(2.9). If $F$ has characteristic $p, U$ is an indecomposable $F G$-module and $H \leq O_{p}, Z(G)$, then $U_{H}$ is homogeneous.

If $U$ is a principal indecomposable $2\left(p^{\alpha}\right)$-module and $H \leq O_{p^{\prime}} z(G)$, then $\left(p^{\alpha-1} U\right)_{H}$ is homogeneous by (2.8) and (2.9). But if $0 \leq \beta<\alpha$ then $p^{\beta} U / p^{\beta+1} U$ and $p^{\alpha-1} U$ are isomorphic $z\left(p^{\alpha}\right) G$-modules, an isomorphism being given by

$$
p^{\beta} u+p^{\beta+1} U+p^{\alpha-1} u
$$

Thus $U_{H}$ is homogeneous:

(2.10). If $U$ is a principal indecomposable $Z\left(p^{\alpha}\right) G$-module and $H \leq O_{p}, Z(G)$, then $U_{H}$ is homogeneous.

The next result is a simple consequence of $I, 4.4$ of [7].

(2.11). If $G$ is a $p^{\prime}$-group and $U$ is a non-zero homogeneous $z\left(p^{\alpha}\right) G$-module then $U$ is faithful if and only if any composition factor of 
$U$ is faithful.

We shall require the following special case of the subgroup theorem, (44.2) of [5]:

(2.12). If $H \leq G, K \leq Z(G)$ and $U$ is an FK-module, then

$$
\left(U^{G}\right)_{H} \cong\left(\left(U_{H \cap K}\right)^{H}\right)^{\oplus \mathcal{K}},
$$

where $k$ is the index of HK in $G$.

It is easy to check:

(2.13). If $H \leq G$ and $U$ is an FH-module then $C_{G}\left(U^{G}\right) \leq C_{H}(U)$.

The following is effectively proved in the first part of the proof of (63.2) of [5].

(2.14). If $H \leq G$ and $U$ is an FG-module then $U \lesssim\left(U_{H}\right)^{G}$.

If $H \leq G$ then it is easy to see that $F G \cong(E H)^{G}$. Thus, by (2.3), if $U$ is an injective $F H$-module then $U^{G}$ is an injective $F G$-module. If $F$ has characteristic $p$ and $H$ is a $p^{\prime}$-group then every $F H$-module is injective by Maschke's Theorem. Hence

(2.15). If $F$ has characteristic $p, H \leq G, H$ is a $p^{\prime}$-group and $U$ is an FH-module, then $U^{G}$ is an injective FG-moduze.

If $E$ is an extension field of $F$ then clearly $F G \otimes E \cong E G$. Thus, by (2.3),

(2.16). If $E$ is an extension field of $F$ and $U$ is an injective $E G$-module then $U \otimes E$ is an injective EG-module.

(2.17). If $E$ is an extension field of $F$ and $V$ is a principal indecomposable EG-module then, for some principal indecomposable $F G$-module $U, V$ is a direct summand of $U \otimes E$.

We shall also need the following result, to which we have not been able to find any published reference.

(2.18). Suppose $E$ is an extension fiezd of $F$ and $U$ and $V$ are $F G$-modules. Then $U$ and $V$ have a common non-zero direct summand if and only if $U \otimes E$ and $V \otimes E$ have a common non-zero direct summand. 
Proof. If $U$ and $V$ have a common non-zero direct summand then clearly so do $U \otimes E$ and $V \otimes E$. For the converse it suffices to consider the case where $U$ is indecomposable. In this case we prove that $U$ is a direct summand of $V$.

Since $U \otimes E$ and $V \otimes E$ have a common non-zero direct summand there exist elements $\phi$ of $\operatorname{Hom}(U \otimes E, V \otimes E)$ and $\psi$ of $\operatorname{Hom}(V \otimes E, U \otimes E)$ such that $\phi \psi$ is a non-zero idempotent in End $(U \otimes E)$. By (29.3) of [5], we can write

$$
\phi=\sum_{i} \phi_{i} \otimes e_{i}, \psi=\sum_{i} \psi_{j} \otimes e_{j}^{\prime},
$$

where the $e_{i}$ and $e_{j}^{\prime}$ are elements of $E$, the $\phi_{i}$ are elements of $\operatorname{Hom}(U, V)$ and the $\psi_{j}$ are elements of $\operatorname{Hom}(V, U)$. Thus

$$
\phi \psi=\sum_{i, j} \phi_{i} \psi_{j} \otimes e_{i} e_{j}^{\prime} .
$$

Suppose that, for some $i, j$, the element $\phi_{i} \psi_{j}$ is a unit of EndU. Then the element $\phi_{i}$ of $\operatorname{Hom}(U, V)$ has the right inverse $\psi_{j}\left(\phi_{i} \psi_{j}\right)^{-1}$, and it follows that $U$ is a direct summand of $V$ as required. Thus we assume, by way of contradiction, that no $\phi_{i} \psi_{j}$ is a unit. Since $U$ is indecomposable it follows by Fitting's Lemma, I,10.7 of [7], that each $\phi_{i} \psi_{j}$ is nilpotent, and in fact that each element of the right ideal of EndU generated by $\phi_{i} \psi_{j}$ is nilpotent. Consequently, by $v, 2.4$ of [7], the $\phi_{i} \psi_{j}$ generate a nilpotent right ideal in EndU. It follows that the element $\phi \psi$ of $\operatorname{End}(U \otimes E)$ is nilpotent. But this contradicts the fact that $\phi \psi$ is a non-zero idempotent.

\section{Tensor products}

In this section we shall develop a result, (3.5) below, which will be used in the proof of Theorem 1.4. $F$ will be a field and $G$ and $B$ finite groups. If $U$ and $V$ are $E G$-modules then $U \otimes V$ denotes the tensor product of $U$ and $V$, also an FG-module. We shall sometimes call $U \otimes V$ the inner tensor product of $U$ and $V$. If $U$ is an FG-module 
and $V$ is an FH-module then $U \# V$ denotes the outer tensor product of $U$ and $V$ (see (43.1) of [5]): this is an $F(G \times H)$-module. If $n$ is a positive integer we shall write $G^{n}$ for the $n$-th direct power of $G$. The subgroup

$$
\{(g, g, \ldots, g): g \in G\}
$$

of $G^{n}$ will be called the diagonal of $G^{n}$ and has an obvious isomorphism with $G$. If $U$ and $V$ are $F G$-modules then $U \otimes V$ is isomorphic to the restriction of $U \# V$ to the diagonal of $G \times G$ identified with $G$. If $U$ is an $F G$-module then $U^{\# n}$ denotes the n-th outer tensor power of $U$ and is an $F G^{n}$-module. on denotes the $n$-th inner tensor power of $U$. We say that the element $g$ of $G$ acts like a scalar on the FG-module $U$ if $U(f-g)=0$ for some $f \in F$.

If $F$ is algebraically closed, $U$ is an irreducible $F G$-module and $V$ is an irreducible $F H$-module, then $U \# V$ is an irreducible $F(G \times H)$-module, by $\mathrm{V}, 10.3 \mathrm{~b})$ of [7]. Thus, by the remark after the proof of Theorem 2.2 of [6],

(3.1). If $F$ is algebraically closed, $U$ is a principal indecomposable FG-module and $V$ is a principal indecomposable FH-module, then $U \# V$ is a principal indecomposable $F(G \times H)$-module.

It is easy to check (for an arbitrary $F$ ):

(3.2). Let $U$ be an FG-moduze and $V$ an FH-module. The element $(g, h)$ of $G \times H$ acts like a scalar on $U \# V$ if and only if $g$ acts like a scalar on $U$ and $h$ acts like a scalar on $V$.

We shall also need:

(3.3). If $F$ has characteristic $p$, then for each element $g$ of $G \backslash O_{p^{\prime}} Z(G)$, there is a principal indecomposable FG-module on which $g$ does not act like a scalar.

Proof. Suppose that $g \in G$ acts like a scalar on each principal indecomposable $F G$-module. Then, since $F$ has characteristic $p$, there is an integer $k$ prime to $p$ such that $g^{k}$ acts trivially on each principal indecomposable and hence on $F G$. Similarly, if $h \in G$ then 
$g^{-1} h^{-1} g h$ acts trivially on $F G$. Since $F G$ is faithful it follows that $g^{k}=1$ and $g h=h g$. Thus $g \in O_{p^{\prime}} Z(G)$.

Let $U$ be a faithful $F G$-module and suppose that exactly $m$ elements of $G$ act like scalars on $U$. Then, by Theorem 2 of [2], for some positive integer $k, F G$ is a direct summand of

$$
V=v^{\otimes k} \oplus U^{\otimes k+1} \oplus \ldots \oplus U^{\otimes k+m-1}
$$

For any positive integer $d,(F G)^{\oplus d}$ is the module induced from a $d$-dimensional module for the trivial subgroup of $G$. Thus, if $W$ is a d-dimensional $F G$-module, (44.3) of [5] shows that $W \otimes F G \cong(F G)^{\oplus d}$. Thus if $U$ (above) has dimension greater than one, for any $d$ there is an $n$ such that $(F G)^{\oplus d}$ is a direct summand of $U^{\otimes n} \otimes V$ :

(3.4). Suppose that $U$ is a faithful $F G$-module of dimension greater than one on which exactly $m$ elements of $G$ act like scalars. Then for any positive integer $d$ there is a positive integer $k$ such that $(F G)^{\oplus d}$ is a direct summand of

$$
U^{* k} \oplus U^{\otimes k+1} \oplus \ldots \oplus U^{\otimes k+m-1} \text {. }
$$

The remainder of this section will be devoted to a proof of the following result.

(3.5). Let $F$ have characteristic $p$ and suppose that $I_{H}=O_{p}, Z(G) \neq G$. Suppose that $V$ is an $F G$-module such that $V_{H}$ is homogeneous. Then, for some $n$ and some principal indecomposable $E G^{n}$-module $W, V \leqslant W_{G}$ where $G$ is identified with the diagonal of $G^{n}$.

Proof. It is sufficient to prove the result in the case where $V$ is infective - otherwise replace $V$ by $\left(V_{H}\right)^{G}$, using (2.14), (2.15) and (2.12). Clearly we may also assume that $V$ is non-zero.

First suppose that $F$ is algebraically closed. In this case the outer tensor product of $n$ principal indecomposable $F G$-modules is, by (3.1), a principal indecomposable $F G^{n}$-module. Thus it suffices to show 
that $V$ is isomorphic to a submodule of some inner tensor product of principal indecomposable $F G$-modules.

Since $F$ is algebraically closed and $H$ is abelian, every irreducible $F H$-module is one-dimensional. Since $H$ is a $p^{\prime}$-group, every $F G$-module is completely reducible by Maschke's Theorem. Therefore every element of $H$ has scalar action on every homogeneous FH-module. By (2.9), every element of $H$ has scalar action on every principal indecomposable FG-module. Thus, by (3.2), every element of $H$ has scalar action on any inner tensor product of principal indecomposable $F G$-modules. Let $U_{1}$ be the inner tensor product of representatives of all the isomorphism types of principal indecomposable $F G$-modules and let $U_{2}=U_{1}^{\otimes L}$ where $Z$ is the exponent of $H$. Then every element of $H$ has scalar action on $U_{1}$ and hence acts trivially on $U_{2}$. On the other hand, by (3.3) and (3.2) no element of $G \backslash H$ acts like a scalar on $U_{2}$.

Since $V$ is injective we can write

$$
V \cong \bigoplus_{i=1}^{r} V_{i}^{\oplus d(i)},
$$

where the $V_{i}$ are pairwise non-isomorphic principal indecomposable $F G$-modules, the $d(i)$ are positive integers, and $r \geq 1$ since $V$ is non-zero. Since $V_{H}$ is completely reducible and homogeneous it is a direct multiple of some irreducible $F H$-module $P$. Thus, for each $i$, $\left(V_{i}\right)_{H}$ is also a direct multiple of $P$. Let $N=C_{H}(P)$ and let $U=V_{1} \otimes U_{2}$. Then $U_{H}$ is a direct multiple of $P$. No element of $G \backslash H$ acts like a scalar on $U$ and so $N=C_{G}(U)$. We now regard $V$, the $V_{i}$ and $U$ as $F(G / N)$-modules and $P$ as an $F(H / N)$-module. $U$ is a faithful $F(G / N)$-module and $H / N$ is the subgroup of $G / N$ consisting of those elements which act like scalars on $U$. Since $H \neq G, U$ has dimension greater than one. Let $d$ be the maximum of the $d(i)$ and $m$ the order of $H / N$. Then, by (3.4), there is a $k$ such that $(F(G / N))^{\oplus d}$ is a direct summand of 


$$
U^{\otimes k} \oplus v^{\otimes k+1} \oplus \ldots \oplus U^{\otimes k+m-1}
$$

The restriction of $U^{\otimes k+i}$ to $H / N$ is a direct multiple of $P^{\otimes k+i}$. Thus for exactly one $j, 0 \leq j \leq m-1,\left(U^{\otimes k+j}\right)_{H / N}$ is a direct multiple of $P$. By (2.5) each $V_{i}$ is a principal indecomposable $F(G / N)$-module; and $\left(V_{i}\right)_{H / N}$ is a direct multiple of $P$. Consequently, for each $i, V_{i}^{\oplus d}$ is a direct summand of $v^{\otimes k+j}$. Thus $V$ is a direct summand of $U^{\otimes k+j}$, which is an inner tensor product of principal indecomposable $F G$-modules as required.

Now let $F$ be arbitrary (of characteristic $p$ ) and $E$ the algebraic closure of $F$. Since $V$ is injective we can write

$$
V \cong \underset{i}{\oplus} v_{i}^{\oplus d(i)}
$$

where the $V_{i}$ are pairwise non-isomorphic principal indecomposable $F G$-modules and the $d(i)$ are positive integers. $V_{H}$ is homogeneous and completely reducible, so it is a direct multiple of some irreducible FH-module $P$. Let $P^{\prime}$ be an indecomposable direct summand of $P \otimes E$. Then $P^{\prime}$ is irreducible by Maschke's Theorem. For each $i, \quad\left(V_{i} \otimes E\right)_{H}$ is a direct multiple of $P \otimes E$, and, by (2.16), $V_{i} \otimes E$ is an injective EG-module. Therefore, by (2.3) and (2.9), $V_{i} \otimes E$ has a principal indecomposable direct summand $V_{i l}$ whose restriction to $H$ is a direct multiple of $P^{\prime}$. Suppose that, for each $i$,

$$
V_{i} \otimes E \cong V_{i 1}^{\oplus k(i)} \oplus V_{i 2} \text {, }
$$

where $V_{i 1}$ is not a direct summand of $V_{i 2}$. By the first part we can choose $n$ and a principal indecomposable $E G^{n}$-module $W^{\prime}$ such that

$$
\bigoplus_{i} V_{i 1}^{\oplus d(i) k(i)}
$$

is a direct summand of $\left(W^{\prime}\right)_{G}$ - where $G$ is identified with the diegonal 
of $G^{n}$. By (2.17) there is a principal indecomposable $F G^{n}$-module $W$ such that $W^{\prime}$ is a direct summand of $W \otimes E$. Thus

$$
\oplus v_{i 1}^{\oplus d(i) k(i)}
$$

is a direct summand of $(W \otimes E)_{G} \cong W_{G} \otimes E$.

Suppose that

$$
W_{G} \simeq\left(\oplus V_{i}^{\oplus Z(i)}\right) \oplus w^{\prime \prime}
$$

where no $V_{i}$ is a direct summand of $W^{\prime \prime}$. Then

$$
W_{G} \otimes E \equiv\left\{\Theta\left(V_{i} \otimes E\right)^{\oplus Z(i)}\right\} \oplus\left(W^{\prime \prime} \otimes E\right)
$$

By (2.18), $V_{i l}$ is not a direct summand of $W^{\prime \prime} \otimes E$ or of $V_{j} \otimes E$ for $j \neq i$. Thus

$$
v_{i l}^{\oplus d}(i) k(i)
$$

is a direct summand of

$$
\left(V_{i} \otimes E\right)^{\oplus Z(i)}
$$

Therefore, for all $i, d(i) k(i) \leq l(i) k(i)$ and so $d(i) \leq l(i)$. Thus $V$ is a direct sumend of $W_{G}$ and the proof of (3.5) is complete.

\section{Proof of the theorems}

Throughout this section $\underline{V}$ will be a locally finite variety, $m$ a positive integer, $m>1$, and $\underline{\underline{\mathrm{U}}}=\underline{\underline{\mathrm{A}}} \underline{\underline{\mathrm{V}}}$. We write $F(\infty)=F_{\infty}(\underline{\underline{\mathrm{V}}})$, $G(\infty)=F_{\infty}(\underline{\underline{U}})$ and take $F(\infty), G(\infty)$, to be (relatively) freely generated by $\left\{f_{1}, f_{2}, \ldots\right\},\left\{g_{1}, g_{2}, \ldots\right\}$, respectively. For any positive integer $n$, we write $F(n)=\left\langle f_{1}, \ldots, f_{n}\right\rangle$ and $G(n)=\left(g_{1}, \ldots, g_{n}\right\rangle$. Thus $F(n) \cong F_{n}(\underline{\underline{V}})$ and $G(n) \cong F_{n}(\underline{\underline{U}})$.

Let $\stackrel{M}{=}$ be the class of groups of the form Usplit $H$ where $H \in F(\underline{\underline{V}})$ and $U$ is a faithful principal indecomposable $Z(m) H$-module. We first 
prove that $M(\underline{\underline{U}}) \subseteq s \underline{\underline{M}}$. Suppose $G \in M(\underline{\underline{U}})$. If $\underline{\underline{V}}(G)=1$ then $G \in F(V)$. Since $G$ is monolithic and $Z(m) G$ is faithful there is $a$ faithful principal indecomposable $Z(m) G$-module $U$. Thus $G \in s\left\{U_{\text {split } G\}}\right.$ and $U$ split $\in \underline{\underline{M}}$. Suppose then that $\underline{\underline{V}}(G) \neq 1$. Let $K=G / \underline{\underline{V}}(G)$ and let $U$ be the injective hull of $\underline{\underline{\mathrm{V}}}(G)$ regarded as a $Z(m) K$-module. Since $G$ is monolithic, $\underline{\underline{\mathrm{V}}}(G)$ is a monolithic module. Hence $U$ is monolithic and, by (2.3), $U$ is a principal indecomposable $Z(m) K$-module. Since $\underline{\underline{\mathrm{V}}}(G) \leq U$ we can form a group $G^{*} \geq G$ which is an extension of $U$ by $K$. (Use the same factor set as in the extension $G$ of $\underline{\underline{\mathrm{v}}}(G)$ by $K$. ) By (2.1), $G^{*}$ splits over $U$ and we can write $G^{*}=U_{\text {split } K}$ and regard $K$ as a complement of $U$ in $G^{*}$. Let $N=C_{K}(U)$. Then $N \leq G^{*}$ and $N \cap U=1$. Hence $N \cap G=1$, since $\underline{\underline{V}}(G)$ contains the monolith of $G$. Thus, putting $H=K / N$ and regarding $U$ as a $Z(m) H$-module, $G \in$ s $\{$ split $H\}$. But $U$ is a faithful principal indecomposable $Z(m) H$-module, by $(2.5)$. Thus $M(\underline{\underline{U}}) \subseteq s \underline{\underline{M}}$.

Proof of Theorem 1.2. Let $\underline{M}^{\prime}$ be the class of groups of the form $V$ split $(F(n) / C)$, where $n$ is a positive integer and $V$ is a principal indecomposable $Z(m) F(n)$-module regarded as a module for $F(n) / C$ where $C=C_{F(n)}(V)$. We next show that $\underline{\underline{M} \subseteq Q \underline{S M}^{\prime}}$. Suppose that UsplitH $\in \underline{\underline{M}}$. Take $n$ so that there is an epimorphism $\theta: F(n) \rightarrow H$ and regard $U$ as a $Z(m) F(n)$-module, via $\theta$. Let $V$ be the injective hull of $U$. Since $U$ is monolithic by (2.4), $V$ is monolithic. Thus, by (2.3), $V$ is a principal indecomposable $Z(m) F(n)$-module. Let $C=C_{F(n)}(V)$. Then $C \leq \operatorname{ker} \theta$, since $U$ is a faithful $Z(m) H$-module, and so $U$ split $H$ is a section of Vsplit $(F(n) / C)$. Thus $\underline{\underline{M}} \subseteq Q \mathrm{MM}^{\prime}$.

Since

$$
\mathrm{C}(\underline{\underline{\mathrm{U}}}) \subseteq M(\underline{\underline{U}}) \subseteq \underline{\underline{M}} \subseteq Q \underline{\underline{M}} \underline{\underline{I}}^{\prime}
$$

and $C(\underline{\underline{U}})$ generates $\underline{\underline{U}}$, Theorem 1.2 will follow if we can show that $\underline{\underline{M}}^{\prime} \subseteq S(\underline{\underline{U}})$. Suppose then that $\underline{\underline{D}}$ is a generating class for $\underline{\underline{U}}$ and $G \in M^{\prime}$,

$$
G=\operatorname{Vsplit}(F(n) / C)
$$

We shall show that $G \in Q \mathrm{QD}$.

Let $U=\underline{\underline{V}}(G(n+l))$. Then there is an isomorphism $\xi: U G(n) / U \rightarrow F(n)$ 
in which $\left(U_{g_{i}}\right) \xi=f_{i}, i=1,2, \ldots, n$. Since $U \in \stackrel{A}{\Rightarrow}$, we may regard $U$ as a $Z(m) F(n)$-module, via $\xi$. Let $e$ be the exponent of $\underline{\underline{v}}$. Then

$$
g=\left(g_{n+1}\right)^{e} \in U
$$

We now prove that $g$ generates a regular submodule of $U$. Let $W$ be a regular $Z(m) F(n+1)$-module generated by an element $\omega$, and regard $H=W s p l i t F(n+1)$ as the semidirect product of subgroups $W$ and $F(n+1)$. Since $H \in \underline{\underline{U}}$ there is a homomorphism $\phi: G(n+1)+H$ in which $g_{i} \phi=f_{i}$, $i=1,2, \ldots, n$, and $g_{n+1} \phi=w f_{n+1}$. Thus writing $w$ additively,

$$
g \phi=\sum_{i=0}^{e-1} w^{f_{n+1}^{i}} .
$$

Since $w$ generates the regular $2(m) F(n+1)$-module $W$ it is clear that $g \phi$ generates a regular submodule of $W_{F(n)}$. It follows that $g$ generates a regular submodule of $U$.

Since $U$ contains a regular $Z(m) F(n)$-module we may take $V \leq U$. Thus, by (2.4), $\sigma V$ is a minimal normal subgroup of $U G(n)$. Since $\underline{\underline{D}}$ generates $\underline{\underline{U}}$ and $G(n+1)$ is relatively free in $\underline{\underline{U}}$, there is a group $D$ in $\underline{\underline{D}}$ and a homomorphism $\psi: G(n+1) \rightarrow D$ in which $\sigma V \ddagger \operatorname{ker} \psi$. Let $J=(\operatorname{ker} \psi) \cap U G(n)$. Then $\sigma V \cap J=1$ and consequently $V \cap J=1$. Let $N$ be a normal subgroup of $U G(n)$ containing $J$ and maximal subject to $V \cap N=1$. Then $U G(n) / N \in Q s \underline{\underline{D}}$. But, by (2.2),

$$
U G(n) / N \cong V_{\text {split }}(F(n) / C) \text {. }
$$

This completes the proof of Theorem 1.2.

Proof of Theorem 1.4. From now on we take $m=p^{\alpha}$, where $\alpha$ is a positive integer, and assume that $\underline{\underline{V}}$ is not abelian of exponent prime to $p$. Also we put

$$
\underline{W}=\underline{V}_{p}=\operatorname{var} F(\infty) / O_{p^{\prime}} Z(F(\infty)) .
$$

Thus $\underline{\underline{\mathrm{W}}}$ is non-trivial and, for any group $H$ of $\underline{\underline{\mathrm{V}}}, \underline{\underline{\mathrm{W}}}(H) \leq O_{p^{\prime}} Z(H)$. We shall call a finite group $p$-special if it is an extension (necessarily split) of an abelian $p$-group $A$ by a cyclic $p^{\prime}$-group $B$ such that, if 
$A$ is regarded as a $B$-module, $A$ is homogeneous with faithful composition factors. It is straightforward to check that the class of $p$-special groups is section closed.

Let $\underline{\underline{S}}$ denote the class of groups $G$ of $F(\underline{\underline{U}})$ such that $\underline{\underline{W}}(G)$ is $p$-special. Then it is easy to see that $\underline{\underline{S}}$ is also the class of groups of $F(\underline{U})$ which are an extension of a group $U \in \stackrel{A}{\Rightarrow}$ (recall $\left.m=p^{\alpha}\right)$ by a group $H \in \underline{\underline{\mathrm{V}}}$ such that $\underline{\underline{W}}(H)$ is cyclic and, if $U$ is regarded as a $W(H)$-module, it is homogeneous with faithful composition factors. Theorem 1.4 is equivalent to the statement $S(\underline{\underline{U}})=\underline{\underline{S}}$. Since the proof of Theorem 1.2 shows that $S(\underline{\underline{U}})=Q \underline{\underline{M}}$, to prove Theorem 1.4 it suffices to prove that $\underline{\underline{S}}=\mathrm{QSM}$.

We first prove that $Q \underline{M} \subseteq \underline{\underline{S}}$. Let $G \in \underline{\underline{M}}, G=U$ split $H$. Since $\underline{W}(H) \leq O_{p^{\prime}} Z(H), U_{\underline{W}(H)}$ is homogeneous by (2.10). It is faithful since $U$ is faithrul. Thus, by (2.11), $U_{\underline{W}(H)}$ has faithful composition factors. Since $W(H)$ is abelian and has a faithful, irreducible $Z(p)$-module it is cyclic. Thus $G \in \underline{\underline{S}}$ by the second characterization of $\underline{\underline{S}}$. If $G \in \underline{\underline{S}}$ and $H \in Q S\{G\}$, then $\underset{W}{W}(H) \in Q S\{\underline{\underline{W}}(G)\}$. Thus $\underline{S}$ is section closed since the class of $p$-special groups is section closed. Hence $Q \underline{\underline{M}} \subseteq \underline{\underline{S}}$.

To complete the proof of Theorem 1.4 we must show that $\underline{\underline{\mathrm{S}}} \subseteq \mathrm{QS \underline { \underline {M } }}$. Suppose then that $G \in \underline{\underline{S}}$ : we shall show that $G \in Q S \underline{\underline{M}}$. Using the second description of $\underline{\underline{S}}, G$ is an extension of a group $U \in \underline{A}_{m}$ by a group $H \in \mathrm{V}$ such that $\underline{\underline{\mathrm{W}}}(H)$ is cyclic and, if $U$ is regarded as a $\mathrm{W}(H)$-module, $U$ is homogeneous with faithful composition factors. We first consider the case where $U=1$. Since $\underline{\underline{W}}(H)$ is a cyclic $p^{\prime}$-group there is a faithful irreducible $Z(p) \underline{\underline{W}}(H)$-module $P$, and $G=H$ is obviously isomorphic to a subgroup of $\left(P^{H}\right) \operatorname{splitH}$. But $\left(P^{H}\right)_{\underline{\underline{W}}(H)}$ is homogeneous with faithful composition factors by (2.12). Thus we may now assume that $U \neq 1$.

Let $U^{*}$ be the injective hull of $U$ as $Z(m) H$-module. Then, since $U \leq U^{*}$, we can form an extension $G^{*} \geq G$ of $U^{*}$ by $H$. It suffices to show that $G^{*} \in Q \mathrm{MM}$. But, by (2.1), $G^{*} \cong U^{*} \operatorname{split} H$. Let $V=\sigma U=\sigma U^{*}$. Then $V$ is a non-zero $Z(p) H$-module whose restriction to $\underline{\underline{W}}(H)$ is homogeneous and faithful. We now apply the following lemma, whose proof we 
leave till last.

(5.1). Let $H \in F(\underline{\underline{V}})$ and let $V$ be a non-zero $2(p) H$-module whose restriction to $\mathrm{W}(H)$ is faithful and homogeneous. Then there are finite groups $K, L, M$ of $\mathrm{V}, K \geq L \geq M$, such that $H \cong L / M$ and, if $V$ is regarded as a $Z(p) L$-module, then $V \leqslant W_{L}$ for some faithful principal indecomposable $Z(p) K-$ module $W$.

Let $K, L, M$ and $W$ be as in the conclusion of (5.1). Let $W^{*}$ be the injective hull of $W$ as a $Z(m) K$-module. Since $\sigma W^{*}=\sigma W, W^{*}$ is monolithic by (2.4). Thus, by (2.3), it is a principal indecomposable $Z(m) K$-module. Also, $W^{*}$ is clearly faithful, since $W$ is faithful. Thus $W^{*}$ splitK is in $\underline{\underline{M}}$ and so $\left(W^{*}\right)_{L}$ split $L$ is in $\underline{\underline{M}}$. Since $V \lesssim W_{L}$, there is a monomorphism $\theta: V \rightarrow\left(W^{*}\right)_{L}$. But $\left(W^{*}\right)_{L}$ is injective by $(2.6)$. Consequently there is a homomorphism $\phi: U^{*} \rightarrow\left(W^{*}\right)_{L}$ whose restriction to $V$ is $\theta$. Since $V=\sigma U^{*}, \phi$ is a monomorphism. Thus $U^{*} \operatorname{split} L$ is in $s \underline{\underline{M}}$ and $U^{*} \mathrm{split} H$ is in $Q s \underline{\underline{M}}$. This completes the proof, apart from the proof of (5.1).

Proof of (5.1). In this proof all modules are over $Z(p)$. Choose $n$ so that $H$ is isomorphic to a factor group $F(n) / N$ of $F(n)$ and write $Y=\underline{W}(F(n))$ and $Z=o_{p^{\prime}} z(F(n+1))$. Since $\underline{\mathrm{V}}$ is not abelian of exponent prime to $p$, and $n+1 \geq 2, Z \neq F(n+1)$. Suppose $z \in Z \cap F(n)$. Then, for some integer $k$ prime to $p, z^{k}=1$ and $z f_{n+1}=f_{n+1} z$. Since $z$ is a word in $f_{1}, f_{2}, \ldots, f_{n}$ only, it follows that $z \in O_{p}, Z(F(\infty))$. Thus $z \in Y$ and $Z \cap F(n) \leq Y$. But $Y \leq \underline{\underline{w}}(F(n+1)) \leq Z$ and so $2 \cap E(n)=Y$.

$$
\text { Since } \begin{aligned}
H \cong F(n) / N \text { we can regard } V \text { as an } F(n) \text {-module, and, since } \\
\\
\underline{\underline{W}}(F(n) / N)=Y N / N,
\end{aligned}
$$

$V_{Y}$ is homogeneous and $C_{Y}\left(V_{Y}\right) \leq N$. For a $p^{\prime}$-group a homogeneous module is a direct multiple of a principal indecomposable module, by Maschke's Theorem. Thus, by (2.7), there is a homogeneous z-module $U$ such that $v_{Y} \leqslant U_{Y} \cdot$ By (2.12), $\left(U^{F(n+1)}\right)_{Z}$ is a direct multiple of $U$, and so is 
homogeneous. Thus, by (3.5), there is a positive integer $r$ and $a$ principal indecomposable module $W$ for $(E(n+1))^{r}$ such that, identifying $F(n+1)$ with the diagonal of $(F(n+1))^{r}$,

$$
U^{F(n+1)} \lesssim W_{F(n+1)}
$$

Therefore

$$
\left(U^{F(n+1)}\right)_{F(n)} \lesssim W_{F(n)}
$$

By $(2.14)$,

$$
V \lesssim\left(V_{Y}\right)^{F(n)} \lesssim\left(U_{Y}\right)^{F(n)}=\left(U_{Z n F(n)}\right)^{F(n)}
$$

and by (2.12),

$$
\left(U_{Z \cap F(n)}\right)^{F(n)} \lesssim\left(U^{F(n+1)}\right)_{F(n)}
$$

Therefore $V \leqslant W_{F(n)}$.

Now let

$$
C=C_{(F(n+1))^{p^{(W)}}}
$$

Then

$$
C \cap F(n+1) \leq C_{F(n+1)}\left(U^{F(n+1)}\right) \leq 2,
$$

by (2.13). Therefore $C \cap F(n) \leq Z \cap F(n)=Y$. But

$$
C \cap Y=c_{Y}\left(W_{Y}\right) \leq C_{Y}\left(V_{Y}\right) \leq N \text {, }
$$

and so $C \cap F(n) \leq N$. Take

$$
K=(F(n+1))^{r} / C, L=F(n) C / C, M=N C / C .
$$

Then $W$ is a faithful principal indecomposable $K$-module by (2.5). Also

$$
L / M \cong F(n) C / N C \cong F(n) /(E(n) \sim N C) \cong H,
$$

since $F(n) \cap N C=N(C \cap F(n))=N$. (5.1) follows. 


\section{References}

[1] Warren Brisley and L.G. Kovács, "On soluble groups of prime-power exponent", Bull. Austral. Math. Soc. 4 (1971), 389-396.

[2] R.M. Bryant and L.G. Kovács, "Tensor products of representations of finite groups", (to appear).

[3] P.J. Cossey, "On varieties of A-groups", Ph.D. thesis, Australian National University, 1966.

[4] John Cossey, "Critical groups and the lattice of varieties", Proc. Amer. Math. Soc. 20 (1969), 217-221.

[5] Charles W. Curtis; Irving Reiner, Representation theory of finite groups and associative algebras (Pure and Applied Mathematics, XI. Interscience [John Wiley \& Sons], New York, London, 1962).

[6] Burton Fein, "Representations of direct products of finite groups", Pacific J. Math. 20 (1967), 45-58.

[7] B. Huppert, Endliche Gmuppen I (Die Grundlehren der mathematischen Wissenschaften, Band 134. Springer-Verlag, Berlin, Heidelberg, New York, 1967).

[8] L.G. Kovács and M.F. Newman, "On critical groups", J. Austral. Math. Soc. 6 (1966), 237-250.

[9] Hanna Neumann, Varieties of groups (Ergebnisse der Mathematik und ihrer Grenzgebiete, Band 37. Springer-Verlag, Berlin, Heidelberg, New York, 1967).

Department of Mathematics, Institute of Advanced Studies, Australian National University, Canberra, ACT. 